\title{
A new azo dye for the selective detection of glycine
}

Bharti Koshti $^{[\mathrm{a}]}$, Vivekshinh Kshtriya ${ }^{[\mathrm{a}]}$, Nidhi Gour ${ }^{[\mathrm{a}]^{*}}$

[a] Department of Chemistry, Indrashil University, Kadi, Mehsana, Gujarat, India; Email: nidhi,gour@indrashiluniversity.edu.in

\begin{abstract}
In this manuscript, we report the synthesis of new azo dye (E)-4-((5-methoxythiazolo[4,5b]pyridine-2-yl)diazenyl)N-phenylaniline (BK2). This new azo dye revealed a pink color in the solution. Notably, this dye selectively detects only glycine among all the amino acids and a visual color change from light pink to purple can be assessed. Hence, the new azo dye finds application as colorimetric chemosensor for the rapid, selective and sensitive detection of glycine (Gly). The dye can be potentially used for the detection of high levels of glycine in the serum caused due to hyperglycinaemia.
\end{abstract}

\section{Keywords}

Azo dye, Glycine, Colorimetric chemosensor, Amino acid

\section{Introduction}

Nowadays, azo dyes are widely used in the field of chemistry. They are also used in the field of pharmaceutical, cosmetic, ${ }^{1}$ and textile industries. ${ }^{2-4}$ Azo dyes are easily synthesized by the process of diazotization..$^{5-7}$ Approximately $70 \%$ dyes are azo dyes which are used in the industry. ${ }^{8}$ The color of the azo dyes are classified according to the number of azo groups which are present in the molecule. A number of heterocyclic azo dyes have been used in the medicinal application.

Various methods have also been used for amino acid sensing which include the colorimetric and fluorescence detection of natural amino acid. In this context, copper complex of the quinacridone ligand is used as a fluorescent sensor for the detection of amino acids L-methionine (Met) and L-leucine (Leu) ${ }^{9}$. In other study a water soluble fluorescein-based ligand forming a non-fluorescent complex with $\mathrm{Cu}^{2+}$ is used for the 
sensing of amino acid. ${ }^{10}$ Various literature report suggest application of azo dyes for selective sensing of amino acid, ${ }^{11} 12$

Our group has been interested in assessing the self-assembling properties of amino acids, ${ }^{13}$, 14 its sensing, peptide, oligonucleotide, biopolymers, and heterocyclic compounds. ${ }^{15}$ Recently, we also reported a colorimetric detection of $\mathrm{Cu}^{2+}$ and lactic by using disaggregation-induced emission mechanisms. ${ }^{16}$ In other study we also reported a new azo dye based sensor for selective and sensitive detection of $\mathrm{Cu}(\mathrm{II}), \mathrm{Sn}(\mathrm{II})$, and Al(III) ions. ${ }^{17}$ Hence, motivated by our previous studies we were inspired to synthesize a new azo dye that could efficiently be used for the colorimetric detection of amino acids.. In this study, we report a rapid, highly sensitive and selective chemosensor for the detection of glycine employing azo dyes (E)-4-((5-methoxythiazolo[4,5-b]pyridine-2yl)diazenyl)N-phenylaniline (BK2).

\section{Result and discussion:}

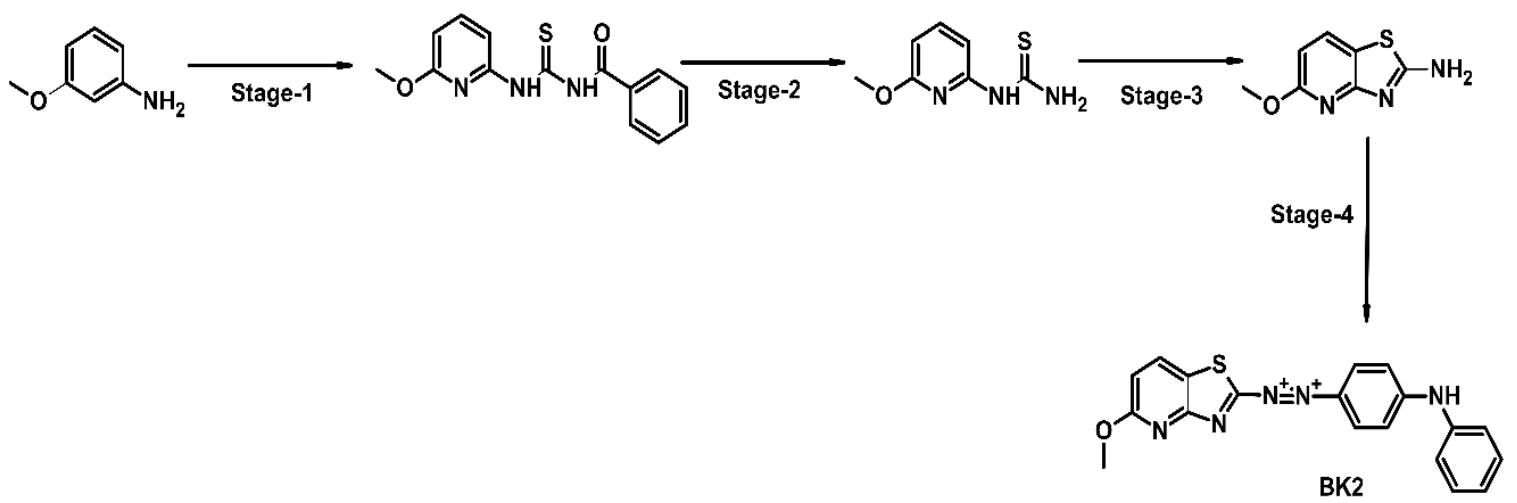

Scheme 1: Schematic representation of synthesis of BK2 
BK2 was synthesized via Scheme 1. In the first step the reaction between benzoyl chloride and ammonium thiocyanate leads to the formation of benzoyl isothiocyanate. It is followed by the condensation reaction between 2-amino-6-methoxy pyridine and benzoyl isothiocyanate leading to the formation of an acyl thiourea intermediate. In the next step, the hydrolysis of an acyl thiourea intermediate is carried out which leads to the formation of 1-(6-methoxypyridin-2-yl)thiourea. The cyclization reaction further yields 5-methoxythiazolo[4,5-b]pyridin-2-amine. In the final step the synthesized amine was conjugated with diphenylamine through the diazotization reaction which leads to the formation of a new azo dye BK2.

For an excellent chemosensor, a high selectivity and sensitivity of the chemosensor is required. Photophysical properties of BK2 in methanol were investigated upon the addition of all amino acids. As a shown in figure 1, the color changes observed only with the glycine and not with the other amino acids. BK2 forms a light pink to purple color by addition of glycine. Moreover, no obvious change has been observed in the absorption when performing the titration of glycine in the mixture of other amino acids. This fact, indicate a high selectivity of BK2 towards glycine.

Figure 1: The color change of BK2 $(50 \mu \mathrm{M})$ in methanol with $50 \mu \mathrm{M}$ of amino acids.

Amino acid glycine (Gly) plays a crucial role in living system. A mild deficiency of glycine participates in the etiology of metabolic disease. Glycine is an inhibitory neurotransmitter in the central nervous system, including the spinal cord. Therefore, determination of glycine is important in biological system. The excess amount of glycine is also very harmful. Hyperglycinemia is an inborn amino acid disorders which is characterized by the elevated levels of glycine in the blood. Hence, a colorimetric sensors for sensitive detection of glycine through naked eye without using any expensive instrument may be of great utility in diagnosis and treatment.

\section{Experimental section}

\section{Materials and Reagents}


Amino acids including glycine (Gly), cysteine (Cys), histidine (His), tyrosine (Tyr), lysine (Lys), threonine (Thr), methionine (Met), phenylalanine (Phe), tryptophane (Trp), glutamine (Gln), glutamic acid (Glu), isoleucine (Ile), leucine (Leu), alanine (Ala), arginine (Arg), aspartic acid (Asp), valine (Val), serine (Ser), threonine (Thr), and asparagine (Asn) were purchased from SRL (India). 2-amino 6-methoxy pyridine purchased from combi-blocks (USA), Diphenylamine, benzoyl chloride, sodium nitrite and sodium hydroxide were purchased from SD Fine chemical (India). The solvents of analytical grade were purchased from Finar and all the solvents were used without further purification.

\section{General Information}

All organic chemicals and solvents were purchased from SD Fine and Sigma-Aldrich and used without further purification. ${ }^{1} \mathrm{H}$ NMR spectra of chemosensor BK2 were recorded on a Bruker $500 \mathrm{MHz}$ (USA), using TMS as an internal standard, and DMSO as a solvent. Mass spectra were recorded on Bruker-MicrOTOF II (USA). All the amino acids solutions were prepared in the Milli Q water. A $25 \mathrm{mM}$ stock solution of BK2 has been prepared while $10 \mathrm{mM}$ stock solutions of all the amino acids were prepared.

\section{Conclusion}

In conclusion, we have synthesized a new azo dye (E)-4-((5-methoxythiazolo[4,5b]pyridine-2-yl)diazenyl)N-phenylaniline (BK2) which can be used as a highly selective and sensitive colorimetric chemosensor for glycine. Notably, the color of dye changes from pink to purple only on addition of glycine while it remains unaffected by the presence of other amino acids.

\section{Funding sources}

The work was supported by the DST SERB extramural research fund (Project No.EMR/2016/003186) received by Dr. Nidhi Gour.

\section{Author contributions}

Manuscript is written by the contribution of all authors. 


\section{Acknowledgments}

NG and VK greatly acknowledge support from DST and SERB research grant (EMR/2016/003186) for funding and fellowships. BK thanks Indrashil University for funding and infrastructure support.

\section{References}

1. Chen, H., Recent advances in azo dye degrading enzyme research. Current Protein and Peptide Science 2006, 7 (2), 101-111.

2. Benkhaya, S.; M'rabet, S.; El Harfi, A., Classifications, properties, recent synthesis and applications of azo dyes. Heliyon 2020, 6 (1), e03271.

3. Fröse, A.; Schmidtke, K.; Sukmann, T.; Junger, I. J.; Ehrmann, A., Application of natural dyes on diverse textile materials. Optik 2019, 181, 215-219.

4. Ramugade, S. H.; Warde, U. S.; Sekar, N., Azo dyes with ESIPT core for textile applications and DFT study. Dyes and Pigments 2019, 170, 107626.

5. Qiu, J.-K.; Hao, W.-J.; Wang, D.-C.; Wei, P.; Sun, J.; Jiang, B.; Tu, S.-J., Selective sulfonylation and diazotization of indoles. Chemical Communications 2014, 50 (94), 1478214785.

6. Dabbagh, H. A.; Teimouri, A.; Chermahini, A. N., Green and efficient diazotization and diazo coupling reactions on clays. Dyes and pigments 2007, 73 (2), 239-244.

7. Qiu, J.; Xiao, J.; Tang, B.; Ju, B.; Zhang, S., Facile synthesis of novel disperse azo dyes with aromatic hydroxyl group. Dyes and Pigments 2019, 160, 524-529.

8. Yang, Q.; Li, C.; Li, H.; Li, Y.; Yu, N., Degradation of synthetic reactive azo dyes and treatment of textile wastewater by a fungi consortium reactor. Biochemical Engineering Journal 2009, 43 (3), 225-230.

9. Zhou, Y.; Yoon, J., Recent progress in fluorescent and colorimetric chemosensors for detection of amino acids. Chemical Society Reviews 2012, 41 (1), 52-67.

10. Dean, K. E.; Klein, G.; Renaudet, O.; Reymond, J.-L., A green fluorescent chemosensor for amino acids provides a versatile high-throughput screening (HTS) assay for proteases. Bioorganic \& medicinal chemistry letters 2003, 13 (10), 1653-1656.

11. Mohammadi, A.; Khoshsoroor, S.; Khalili, B., Rapid, sensitive and selective detection of arginine using a simple azo-based colorimetric and fluorescent chemosensor. Journal of Photochemistry and Photobiology A: Chemistry 2019, 384, 112035.

12. Zhang, D.; Zhang, M.; Liu, Z.; Yu, M.; Li, F.; Yi, T.; Huang, C., Highly selective colorimetric sensor for cysteine and homocysteine based on azo derivatives. Tetrahedron letters 2006, 47 (39), 7093-7096.

13. Koshti, B.; Singh, R.; Kshtriya, V.; Walia, S.; Bhatia, D.; Gour, N., Amyloid like aggregates formed by the self-assembly of proline and Hydroxyproline. 2021.

14. Gour, N.; Kanth P, C.; Koshti, B.; Kshtriya, V.; Shah, D.; Patel, S.; Agrawal-Rajput, R.; Pandey, M. K., Amyloid-like structures formed by single amino acid self-assemblies of cysteine and methionine. ACS chemical neuroscience 2018, 10 (3), 1230-1239.

15. Gour, N.; Kshtriya, V.; Gupta, S.; Koshti, B.; Singh, R.; Patel, D.; Joshi, K. B., Synthesis and Aggregation Studies of a Pyridothiazole-Based AlEE Probe and Its Application in Sensing Amyloid Fibrillation. ACS Applied Bio Materials 2019, 2 (10), 4442-4455.

16. Kshatriya, V.; Koshti, B.; Pandey, D. K.; Kharbanda, S.; Kanth, C. P.; Singh, D.; Bhatia, D. D.; Gour, N., Sequential and cellular detection of copper and lactic acid by disaggregation and reaggregation of the fluorescent panchromatic fibres of an acylthiourea based sensor. Soft Matter 2021. 
17. Kshtriya, V.; Koshti, B.; Gour, N., A New Azo Dye Based Sensor for Selective and Sensitive Detection of CU (II), Sn (II), and AI (III) lons. 2021. 